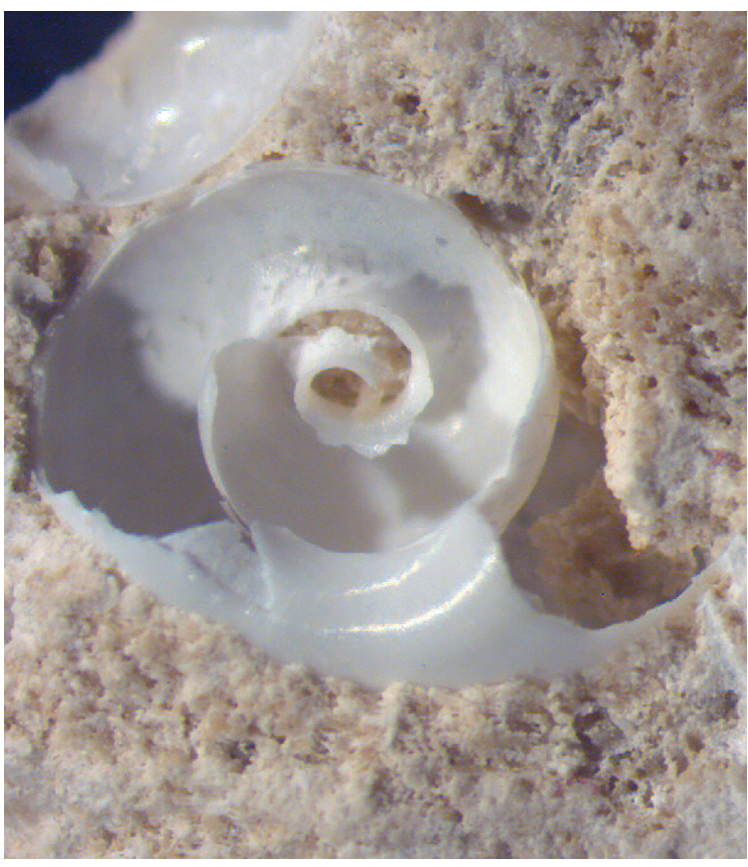

\title{
Evaluación del proceso de carbonatación de nanocales aplicadas a pinturas murales prehispánicas de origen maya
}

An Evaluation of Nanolime Carbonation Process as Applied to pre-Hispanic Mayan Mural Paintings

\author{
Astrid Esquivel Álvarez \\ School of the Art Institute of Chicago (SAIC), Estados Unidos de América \\ astridesquivel7@gmail.com

\section{Laura Filloy Nadal} \\ Museo Nacional de Antropología (MNA), \\ Instituto Nacional de Antropología e Historia (INAH), México \\ Ifilloy@yahoo.com
}

\section{Resumen}

Desde hace más de una década la nanocal, Ilamada comercialmente Nanorestore ${ }^{\circledR}$-que sintetiza el Consorzio Interuniversatario per lo Sviluppo del Sistemi a Grande Interfase ([CSGI], Centro de Interuniversitario de Estudios de Sistemas de Gran Interfase Università degli Studi di Firenze [Unifi], Universidad de Florencia, Italia)—, se utiliza en México para la consolidación de pinturas murales prehispánicas con problemas de disgregación y pulverulencia en su capa pictórica. La efectividad de este nano-material depende, no obstante, de una serie de variables que suelen no tomarse en consideración. Esta INVESTIGACIÓN evalúa los diversos factores tanto intrínsecos como extrínsecos a las pinturas murales involucradas en el proceso de carbonatación de Nanorestore ${ }^{\circledR}$, con el propósito de que, en futuras intervenciones, el tratamiento se encamine hacia resultados óptimos.

\section{Palabras clave}

pintura mural; disgregación; capa pictórica; consolidación; Nanorestore ; carbonatación

\section{Abstract}

For over a decade, a nano-lime known commercially as Nanorestore - which is synthetised by el Consorzio Interuniversatario per lo Sviluppo del Sistemi a Grande Interfase ([CSGI], Centre for Colloid and Surface Science, Università degli Studi di Firenze [UniFi], Florence University, Italy)— has been used in Mexico for the consolidation of pre-Hispanic mural paintings with a pictorial layer that suffers from disaggregation and dustiness. Nevertheless, the effectiveness of this nano-material depends on a series of variables that are oftentimes ignored. This RESEARCH evaluates the intrinsic and extrinsic factors innate to mural paintings that bear upon the carbonation process of Nanorestore ${ }^{\circledR}$ with the purpose of leading future treatments towards optimal results. 


\section{Key words}

mural paintings; dustiness; pictorial layer; consolidation; Nanorestore ${ }^{\circledR}$; carbonation

Introducción: el problema de la consolidación de sustratos calcáreos

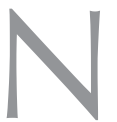
o es novedad que la consolidación ${ }^{1}$ constituye un paso fundamental en las intervenciones para la conservación de pinturas murales que presentan falta de cohesión, disgregación y/o pulverulencia ${ }^{2}$ en su capa pictórica. En el caso de murales elaborados a base de cal, para un profesional de la conservación-restauración sería convencional proponer que un material idóneo para llevar a cabo su consolidación es el agua de cal, ya que al estar compuesta de partículas de hidróxido de calcio en agua, ésta penetraría en el sustrato debilitado por el tiempo y, al cristalizar —o carbonatar-, formaría una nueva matriz cristalina con idénticas propiedades que el material original.

Desafortunadamente, hay que señalar que el agua de cal no se desempeña como un consolidante enteramente efectivo: ello se debe a que la baja concentración de la solución acuosa saturada, equivalente a 1.7 g de hidróxido de calcio por litro de agua (D'Armada 2012:64), no sólo obligaría a repetir el procedimiento casi de manera indefinida para restablecer la cohesión, sino que además implicaría el ingreso de grandes cantidades de agua en la estructura, lo que a su vez provocaría la difusión de sales y el arrastre de material arcilloso.

Gracias a varios experimentos recientemente se ha logrado suspender en medios alcohólicos partículas nanométricas de hidróxido de calcio, comúnmente llamadas nanocales (Ambrosi 2001a, 2001b; Baglioni 2006a; Baglioni et al. 2006b, 2009; Dei 2004, 2005; Giorgi et al. 2000, 2002, 2010), lo que supone grandes ventajas en su empleo en la restauración: estos alcoholes tienen una tensión superficial y una viscosidad lo suficientemente bajas como para garantizar una óptima mojabilidad; su carácter no polar les permite adentrarse a una mayor profundidad en la matriz (entre 2 y $3 \mathrm{~mm}$ ), aparte de que no promueven el arrastre de depósitos salinos (Ambrosi 2001b:69). Asimismo, estas nanopartículas tienen un radio iónico que oscila entre los 50 y los $300 \mathrm{~nm}$, a diferencia del hidróxido de calcio convencional, que suele rondar los 800 nm (D'Armada 2012:64). Esto se traduce en una penetra-

\footnotetext{
${ }^{1}$ La consolidación es un proceso que involucra la aplicación de un material de unión (ligante, aglutinante, cementante) con el fin de mejorar la cohesión entre los materiales que conforman un sustrato y evitar que se desprendan de su soporte (Rodgers 1988:2).

${ }^{2}$ El efecto de disgregación se refiere al desprendimiento de granos individuales, o de conjuntos de granos, donde las partículas del material no conservan la unión y únicamente se mantienen en su sitio gracias a las fuerzas electrostática y de gravedad (cfr. ICOMOS-ISCS 2010:20).
}

ción mayor, en tanto que se evita la posibilidad de que los poros se ocluyan por la aplicación sucesiva del consolidante o de que se forme algún tipo de deposición que altere el color en superficie (Mansoori 2004:3). Finalmente, es factible formular dispersiones con distintas concentraciones, entre 2.5 hasta $10 \mathrm{~g} / \mathrm{L}$, que optimizan las labores de los conservadores.

\section{La experiencia con las nanocales en México}

Desde hace más de un decenio, las nanocales denominadas Nanorestore ${ }^{\circledR}$-mismas que son sintetizadas por el el Consorzio Interuniversatario per lo Sviluppo del Sistemi a Grande Interfase ([CSGI], Centro Interuniversitario de Estudios de Sistemas de Gran Interfase, Università degli Studi di Firenze [Unifi], Universidad de Florencia, Italia - se han utilizado en México gracias a la colaboración de científicos de esa institución en proyectos coordinados por el Instituto Nacional de Antropología e Historia (INAH, México). Las más de las veces el producto se ha empleado en el tratamiento de pinturas murales localizadas en sitios arqueológicos prehispánicos. ${ }^{3}$

Al respecto, llama la atención la escasa producción documental (Baglioni et al. 2006b; Castro et al. 2013; Chelazzi et al. 2013) que define de manera sistemática la metodología empleada o bien ofrezca un análisis profundo de los resultados obtenidos. Asimismo, hemos notado que aunque no hay bibliografía al respecto, entre los profesionales de la conservación abundan las discrepancias acerca de la eficacia de este nano-material. En suma, pareciera, por un lado, que existe una falta de consideración sobre la reactividad de éste y, por otro, que no se ha comprendido cabalmente la incidencia de varios factores, tanto inherentes a la naturaleza de los bienes culturales como externos a ella, que determinen el desempeño del producto, tales como la porosidad, naturaleza del sustrato; medio de dispersión, concentración del consolidante; método, tiempos de aplicación, o humedad relativa (HR) y calidad del aire (Jáidar 2014).

Así, en el $2013^{4}$ nos propusimos, como parte del Laboratorio de Conservación del Museo Nacional de Antropología (LC-MNA) del INAH, Ilevar a cabo una investigación en la que se estudiara de manera aislada el proceso de carbonatación de Nanorestore ${ }^{\circledR}$ y se realizara un experimento de consolidación de fragmentos de pintura mural original, antecedido y sucedido por el análisis minucioso de sus propiedades a escala macroscópica, estructural y morfológica.

\footnotetext{
${ }^{3}$ Destacan pruebas en los estucos policromos de la Subestructura II de la Estructura III de la Zona Arqueológica de Calakmul, Campeche; los murales La Batalla y Templo Rojo de Cacaxtla, Tlaxcala; Los Bebedores, de Cholula, Puebla; la caja de agua, de Tlatelolco, Ciudad de México, y las pinturas del Palacio de los Símbolos Solares, en Mayapán, Yucatán (todos en México) (Grimaldi 2008; García 2008, 2011; Castro 2009).

${ }^{4}$ Véase también Esquivel Álvarez 2014.
} 


\section{Metodología de investigación}

Se fabricaron cuatro cámaras, cada una con un microambiente particular. La primera, tuvo una HR de 30\%, mientras que la de la segunda fue de $80 \%$. Para mantener estas condiciones, en aquella se empleó gel de sílica marca Rhapid $\mathrm{Pak}^{\circledR}$, acondicionada a $30 \%$ de HR, en tanto que para el siguiente caso se usó agua destilada. Estos porcentajes se monitorearon con un termohigrógrafo marca $\mathrm{HOBO}^{\circledR}$. El nivel de $\mathrm{CO}_{2}$ de estas cajas fue el mismo que el del LC-MNA, equivalente a $0.0 \% /$ vol. ${ }^{5}$ A las cámaras 3 y 4 se les asignaron los mismos niveles de HR, pero el doble de contenido de $\mathrm{CO}_{2}$, con el propósito de observar de manera clara el efecto de la calidad y/o composición del aire en el proceso de carbonatación. Para ello se emplearon tabletas dispersables de bicarbonato de calcio marca Alka-Seltzer $^{\circledR}$, cuya medida es de $3.241 \mathrm{~g}$, las cuales liberan al contacto con el agua un estimado de $1.0036 \mathrm{~g}$ de $\mathrm{CO}_{2}$, equivalente a $31 \%$ de su peso original. Así, un par de tabletas fue suficiente para elevarlo hasta $1.0 \% / \mathrm{vol}$. El grado de $\mathrm{CO}_{2}$ en las cámaras se mantuvo estable aún dos días después debido a que aquéllas se sellaron con un filme-barrera de polietileno y nylon aluminizado marca Marvelseal ${ }^{\circledR} 360$.

En cada cámara se introdujo una caja de Petri con muestras de Nanorestore ${ }^{\circledR}$. Aunque este producto se encuentra en dispersiones de isopropanol y n-propanol, se decidió emplear la de etanol, pues presenta una menor viscosidad, un índice de difusión lento —en comparación con el agua- y, al mismo tiempo, una tasa de evaporación menor, por lo que, se cree, tiene mayor capacidad de penetración. Asimismo, con base en la relación entre la naturaleza del disolvente y el tamaño de la partícula, se esperan partículas más pequeñas. La suspensión estuvo a una concentración de $2.5 \mathrm{~g} / \mathrm{L}$ y se inspeccionó a los 5 y 30 días de haberse iniciado el experimento. A estas muestras se las Ilamó NP1, NP2, NP3 y NP4, en función de la cámara en la que se introdujeron.

Junto con los recipientes, se integraron fragmentos de pintura mural prehispánica, procedente del Grupo de las Pinturas de la Zona Arqueológica de Cobá, Quintana $\operatorname{Roo}^{6}$ (México), que fueron consolidadas con Nanorestore $^{\circledR}$. Las muestras se nombraron $M 1, M 2, M 3$ y $M 4$, de acuerdo, también, con sendas cámaras.

Todas las muestras se sometieron a un mismo procedimiento de consolidación: se llevó a cabo un total de doce

\footnotetext{
${ }^{5}$ Se empleó un medidor de gases contaminantes modelo Pac ${ }^{\circledR} 7000$, marca Dräger ${ }^{\circledR}$, equipado con sensores de alta precisión, el cual posee un rango de medición que va desde $0.01 \%$ hasta $5 \% /$ vol.

${ }^{6}$ La pintura mural de Cobá es representativa de la escuela maya de pintura, sin lugar a dudas, una de las más importantes de nuestro país (Folan 1977). Al ser una pintura elaborada en un sustrato de cal (MagaIoni 1996:165-170), sus materiales de manufactura la hacen totalmente compatible con el consolidante aquí evaluado, lo que, en cierto sentido, justifica este tratamiento.
}

aplicaciones consecutivas en cada fragmento a intervalos de tres días, con registro de los resultados a la mitad del proceso (seis aplicaciones) y al final de éste (doce). La cantidad de aplicaciones se determinó con base en la cantidad de éstas hecha a pinturas murales mencionada en la literatura (Grimaldi 2008; García 2008, 2011; Castro 2009) y en las experiencias de las autoras. La dispersión se aplicó exactamente a la misma proporción a la que se hizo el análisis de carbonatación, es decir, 2.5 g/L. Según la metodología propuesta por el CSGI, que se ha empleado en México a lo largo de los años (Grimaldi 2008; García 2008, 2011; Castro 2009), se administró un poco de etanol sobre la superficie para favorecer la penetración del consolidante, y se aplicó la dispersión con ayuda de un pincel de pelo suave sobre un trozo de papel japonés; finalmente, se colocó una papeta de pulpa de celulosa y agua destilada, preparadas empíricamente. Las papetas se sustituyeron conforme se avanzaba en el proceso. Cabe mencionar que hubo una muestra adicional, a la cual nos referiremos como Muestra Control, que no recibió tratamiento alguno, la cual se utilizó como referencia, así como con la finalidad de comparar los cambios obtenidos con la consolidación.

En este trabajo de investigación, las técnicas experimentales jugaron un papel decisivo. Se eligieron asumiendo tanto la necesidad de emplear métodos no destructivos como la riqueza de los datos que se esperaba obtener de cada una de ellas. Etapa por etapa, a la investigación la acompañó una ronda de estudios con el propósito de recopilar la mayor información posible.

La determinación de propiedades morfológicas se hizo empleando un microscopio óptico marca Nikon ${ }^{\circledR}$ SMZ800 con objetivo estándar de 10 x y zoom de 1-6.3 $x$ equipado con una cámara marca Leica ${ }^{\circledR}$ DC3000, propiedad del Laboratorio de Enseñanza de la Escuela Nacional de Conservación, Restauración y Museología (LE/ ENCRYM-INAH, México), así como un microscopio electrónico de barrido (SEM, por sus siglas en inglés) con una microsonda EDS (energy-dispersive X-ray spectroscopy) acoplada, modelo FE SEM JEOL JSM-7600F, operado por el doctor Omar Novelo Peralta, del Instituto de Investigaciones en Materiales (IIM) de la Universidad Nacional Autónoma de México (UNAM, México).

Para la determinación de propiedades estructurales se recurrió a un estudio de difracción de rayos $X$ (por sus siglas en inglés, XRD) modelo Bruker-AXS D8 Advance ${ }^{\circ}$, operado por la maestra Adriana Tejeda Cruz, del Laboratorio de Difracción de Rayos X del IIM-UNAM; los datos obtenidos se procesaron mediante el software Diffrac $\mathrm{Eva}^{\odot}$, cuya base de estándares es el PDF-2 (powder diffraction file) del International Centre of Diffraction Data (ICDD, EUA). Asimismo, se empleó un espectrómetro de infrarrojo por transformada de Fourier (FTIR) modelo Nicolet ${ }^{\circledR} 510 \mathrm{P}$ FT-IR, con un aditamento ATR universal de diamante, propiedad del IIM-UNAM. El procesamiento de datos se llevó a cabo con el software Advantage $\iota^{\circ}$. 
Finalmente, para establecer las propiedades macroscópicas se hicieron pruebas de afinidad superficial derivadas del ángulo de contacto, usando agua destilada. Las imágenes de cada una de ellas se realizaron con una cámara Canon Power Shot ${ }^{\circledR}$ SX40 HS y posteriormente se procesaron en Photo Shop ${ }^{\circledR}$ CS5. También se recurrió a un espectrofotómetro de contacto modelo CM2500d, marca Konika Minolta ${ }^{\circledR}$, propiedad del Laboratorio de Fisicoquímica de la ENCRYM-INAH; un microdurómetro modelo HMV-G, marca Shimadzu ${ }^{\circledR}$, del Laboratorio de Materiales Metálicos Avanzados del IIM-UNAM, de acuerdo con el protocolo establecido en la norma ASTM E384-11E1, ${ }^{7}$ así como el test de la cinta $\operatorname{Scotch}^{\circledR}$, para lo cual se emplearon una balanza analítica marca $\mathrm{And}^{\circledR}$, modelo FR200, también del LE/ENCRYM-INAH, y tiras de cinta adhesiva transparente marca Scotch ${ }^{\circledR}$.

\section{Consideraciones previas}

Antes de proceder al análisis de resultados, es importante tomar en cuenta que el proceso de transformación de las nanopartículas de hidróxido de calcio, también llamado portlandita en carbonato de calcio, comienza cuando éstas se deshidratan y reaccionan con el medio ambiente (Rodríguez-Blanco 2011:265). El carbonato de calcio

\footnotetext{
${ }^{7}$ Método de prueba estándar Knoop y Vickers de dureza de materiales (ASTM 2011).
}

Cámara 1.5 y 30 días:

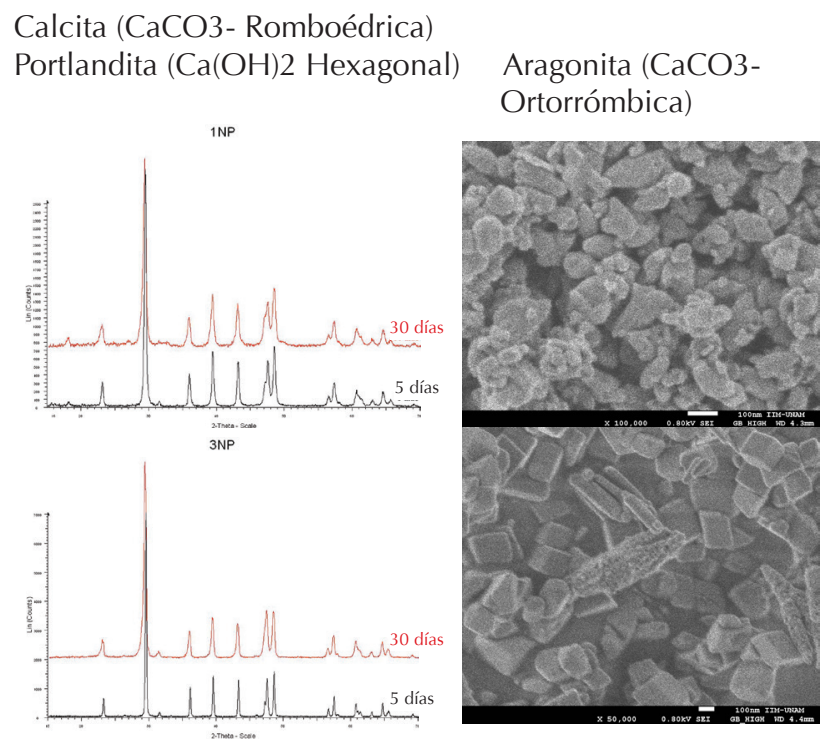

Cámara 3. 5 y 30 días:

Calcita (CaCO3-Romboédrica)

Aragonita (CaCO3-Ortorrómbica) resultante podría adquirir una de las siguientes formas: anhidras o hidratadas, según la presencia o ausencia de moléculas de agua, cuyas estructuras estarán diferenciadas entre sí por los sitios atómicos que ocupan los iones de $\mathrm{Ca}^{2+}$ o del $\mathrm{CO}_{2}^{-}$(Gómez-Villalba et al. 2012b:213).

Se conocen tres fases anhidras, a saber: calcita, aragonita y vaterita, de las cuales la más estable termodinámicamente hablando es la calcita, y la menos, la vaterita, que incluso llega a ser soluble en agua (Dietrich y Skinner 1979:82). Se cree que al inicio del proceso de carbonatación, la portlandita se transforma en aragonita y vaterita, y que posteriormente, gracias al mecanismo de reprecipitación, éstas se convierten en calcita, con lo que se estabilizan por completo y se cierra el proceso de carbonatación (Rodríguez-Blanco 2011:265). Adicionalmente, las formas hidratadas son menos frecuentes que las anhidras y pueden tener una molécula de agua, como es el caso de la monohidrocalcita, o seis, como lo es el de la ikaita (Barthlemy 1997; Friedman 1997).

\section{El experimento de carbonatación}

La combinación de factores como la HR, la cantidad de $\mathrm{CO}_{2}$ en el ambiente, el tiempo y el tipo de disolvente dio resultados muy particulares para cada caso, los cuales se ilustran en la Figura 1.

Como se observa en la Figura 1, en la cámara 1, donde tanto la cantidad de humedad ambiental como de dióxi-

Cámara 2. 5 y 30 días:

$$
\begin{aligned}
& \text { Calcita (CaCO3-Romboédrica) } \\
& \text { Aragonita (CaCO3-Ortorrómbica) }
\end{aligned}
$$
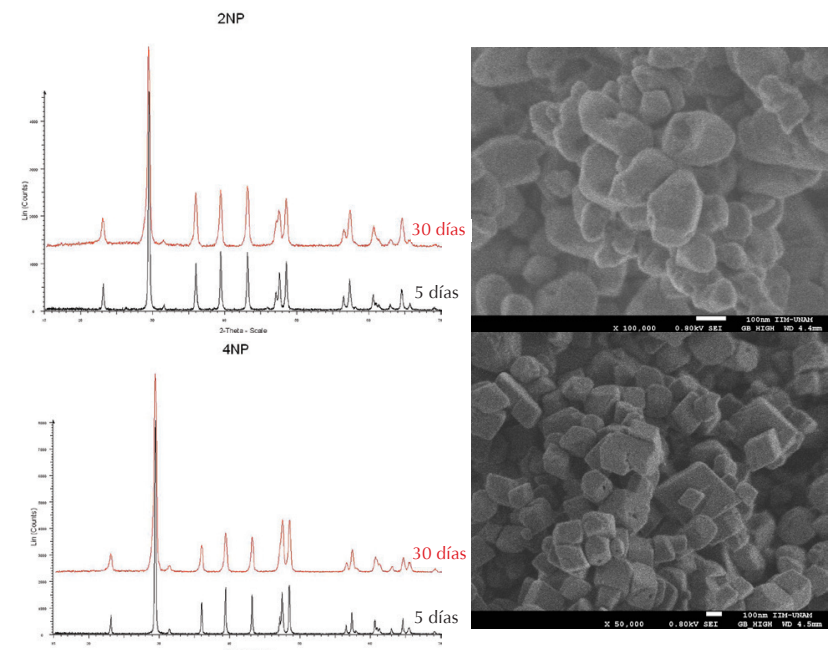

Cámara 4. 5 y 30 días:

Calcita (CaCO3-Romboédrica) 
do de carbono fue pobre, el proceso de carbonatación fue lento, evidenciado por la presencia de portlandita (jcpds $=72-0156, \mathrm{a}=3.585 \AA, \mathrm{c}=4.895 \AA$ ) y una fracción de calcita (jcpds $=01-0862342, \mathrm{a}=4.989 \AA, \mathrm{c}=17.06 \AA$ ) aun después de un mes de exposición en la muestra NP1. Eventualmente, apareció la aragonita (jcpds $=76-0606$, a $=4.959 \AA, b=7.954 \AA, c=5.737 \AA$ ), indicando la reactividad de la portlandita que quedaba (Soldati 2008:577) y la búsqueda de la dispersión por la estabilidad. Los productos obtenidos presentaron una forma ovalada, tamaños de partícula reducidos ( $26.1 \mathrm{~nm}$ en promedio) y niveles de cristalinidad bajos que, en ocasiones, se aglomeraban en forma de racimos; es decir, parece ser que el consolidante tiende a acumularse, como se muestra en la Figura 1. Se piensa que en ambientes de este tipo ocurre una disminución en la tensión superficial de la dispersión y, como consecuencia, se acelera la evaporación del disolvente, con lo que se apresura la nucleación (Gómez-Villalba et al. 2012b:216). Esto evita que las partículas se difundan a sus sitios de energía más bajos, lo cual da origen a partículas más pequeñas y de baja cristalinidad, propensas a la amorfización (López-Arce et al. 2011a:263).

Con la muestra que estuvo dentro de la cámara 2, se observó que la alta HR modificó la concentración de la dispersión, lo que incrementó la tensión superficial y retrasó la evaporación del disolvente (cfr. Gómez-Villalba et al. 2012b:216), pero, al mismo tiempo, aceleró la reacción con el hidróxido de calcio (cfr. López-Arce et al. 2011a: 266). Como resultado, se obtuvieron productos de forma ovalada, ligeramente más ordenados y de mayores dimensiones ( $37.33 \mathrm{~nm}$ en promedio) que los anteriores (Figura 1, cámara 2). Conforme al análisis de DRX, las fases encontradas en esta cámara fueron calcita y aragonita, mismas que aumentaron su cuenta al final del mes: se cree que este fenómeno se debe a la exposición prolongada a la humedad que originó una transición incompleta a la calcita (Ogino 1987:2763).

En la muestra NP3 se identificaron las fases de calcita $y$, en menor cantidad, de aragonita, mismas que indican que el proceso de carbonatación de las nanocales fue más eficiente que en las dos anteriores (Figura 1, cámara 3). Los cristales correspondientes a la calcita tenían formas cúbicas y un tamaño promedio de $43.18 \mathrm{~nm}$, es decir, aún más grandes que los dos casos anteriores. A medida que pasaba el tiempo, los cubos ganaron definición en sus formas. En contraste, los cristales de aragonita siguieron describiendo formas alargadas de extremos angulosos. Se cree que, al no haber agua condensada en superficie - como la había en el caso anterior-, aunado a la alta disponibilidad de $\mathrm{CO}_{2}$, se distribuyó anhídrido carbónico entre los poros de la muestra, lo que agilizó el proceso de nucleación.

Finalmente, en la muestra NP4 la única fase mineralógica fue la calcita. Con seguridad, las condiciones medioambientales de esta cámara hicieron que la disolución de la portlandita $\mathrm{Ca}(\mathrm{OH})_{2}+\mathrm{H}_{2} \mathrm{O} \rightarrow \mathrm{Ca}^{2+}+2 \mathrm{OH}^{-}+$
$\mathrm{H}_{2} \mathrm{O}$ y la disociación de dióxido de carbono $\mathrm{CO}_{2}+\mathrm{H}_{2} \mathrm{O}$

$\rightarrow \mathrm{H}_{2} \mathrm{CO}_{3}$ se llevaran a cabo de manera homogénea y sin interrupciones (Montes-Hernández y Renard 2011:52), hasta que se alcanzó un equilibrio termodinámico entre la calcita y el ambiente. Los cristales obtenidos exhibieron formas cúbicas de alto ordenamiento (Figura 1, cámara 4). Es probable que su aspecto sea una consecuencia de la elevada presión que provocaron el vapor de agua y el $\mathrm{CO}_{2}$ (Montes-Hernández et al 2008:2952). No obstante, al final del experimento se observó que el tamaño de los cristales disminuyó de $54.44 \mathrm{~nm}$ a $22.36 \mathrm{~nm}$, quizá por razón de que el exceso de humedad y de $\mathrm{CO}_{2}$ en la cámara generó un ambiente lo suficientemente ácido como para provocar la disolución del material (Uibu et al. 2010:210-212).

En resumen, mediante este experimento se observó la influencia que tienen tanto la $\mathrm{HR}$ y la cantidad de $\mathrm{CO}_{2}$ en el ambiente como el tiempo y el tipo de disolvente empleados en el fenómeno de carbonatación de las nanopartículas de hidróxido de calcio. En principio, podemos decir que el proceso de carbonatación depende de la cantidad de agua presente en el ambiente, pues ésta impulsa la disolución de la portlandita, así como su transformación en carbonato de calcio, como lo demostraron los resultados de las muestras NP2 y NP4. Los productos obtenidos en estas condiciones presentaron una cristalinidad más elevada, pero también un tamaño de partícula mayor. Por el contrario, los ambientes donde la cantidad de humedad ambiental fue pobre, como los de las cámaras 1 y 3 , se caracterizaron por retardar el proceso. En estos casos, los productos obtenidos fueron pequeños, y sus niveles de cristalinidad, más bajos, además de que hubo porciones de la dispersión que se quedaron reactivas, según lo indicó la presencia de portlandita en la cámara 1 después de un mes de exposición. Por otra parte, la cantidad de dióxido de carbono en el ambiente también fue crucial, pues promovió la carbonatación casi total del material y contrarrestó la aparición de fases metaestables, como la portlandita. La presión que este gas generó junto con el vapor de agua provocó cambios significativos en la morfología de los cristales que quizá podrían incidir en su desempeño como consolidantes.

Finalmente, se observó que la naturaleza del disolvente en el que estaban suspendidas las nanopartículas y la concentración a la que se encontraba la dispersión, así como otras propiedades derivadas de ello, como, por ejemplo, tensión superficial y tasa de evaporación, afectaron significativamente la velocidad de reacción. Adicionalmente, el tiempo se relacionó con las dimensiones de la partícula y, probablemente, con los parámetros de celda, aunque de éstos no se hicieron mediciones.

\section{Desarrollo y resultados del experimento de consolidación}

A continuación se expone el desarrollo del experimento de consolidación, iniciando con la caracterización de la 
pintura mural y su estado de conservación, y concluyendo con sus resultados.

\section{Caracterización de las muestras de pintura mural}

Como se mencionó anteriormente, las cinco muestras que se emplearon en este estudio provinieron del conjunto arqueológico denominado Grupo de las Pinturas, situado en la Zona Arqueológica de Cobá, en el actual estado de Quintana Roo, México. En su momento, Cobá fue uno de los centros urbanos más importantes del noreste de la Península de Yucatán, comparable en tamaño y significado a Chichén Itzá (Folan 1977:32-33). Los fragmentos - que se obtuvieron del Banco de Muestras de la CNCPC-INAH - eran piezas sobrantes de un conjunto de pinturas murales desprendidas en 1975 por un grupo de arqueólogos encabezado por Martine Fettweiss (cfr. Benavides 1981).

Los resultados de la caracterización de las muestras concuerdan plenamente con las observaciones de Diana Magaloni, quien en 1996, como parte de la elaboración de su tesis de maestría, llevó a cabo un estudio sistemático de varias pinturas murales en la zona (MagaIoni 1996). La autora las clasifica como pertenecientes al Grupo Técnico IV, distinguible por un soporte grueso con cargas grandes de formas angulosas, en el cual no existe una diferencia clara entre las capas del aplanado y el enlucido (Figura 2).

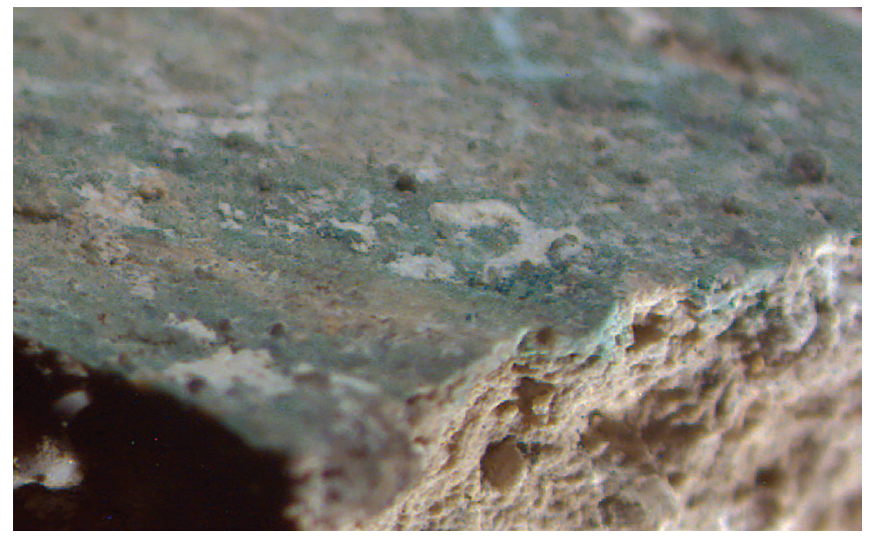

FIGURA 2. Micrografía de la muestra Control Maya (Fuente: Astrid Esquivel Álvarez, 2014. Cortesía: CNCPC-INAH).

Por medio de DRX (Figura 3), se identificaron calcita y aragonita, relacionadas tanto con las cales y rocas empleadas como con los caracoles y conchas (Alton y Mattox 1963:238) insertos en el mortero. Se apreció una contribución muy discreta de cuarzo y no se encontraron rastros de sascab, ${ }^{8}$ la arena calcárea típica del área, presente en las pinturas de Kabah o Chichén Itzá, Yucatán, México (Magaloni 1996:165-170).

\footnotetext{
${ }^{8}$ Sascab o sahacab: en lengua maya, es un suelo blanco derivado de las piedras calizas (Duch-Gary 1991).
}

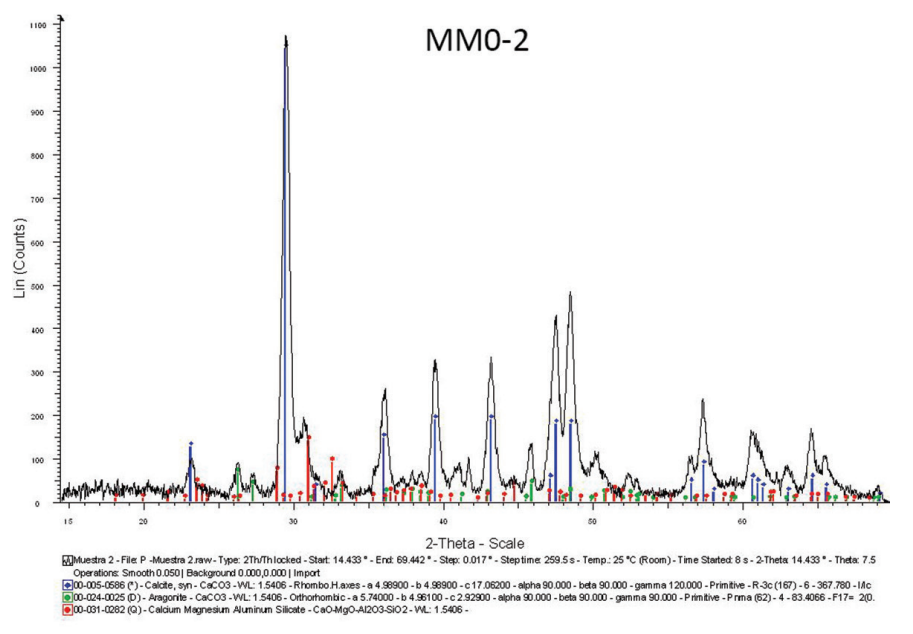

FIGURA 3. Difractograma correspondiente a la muestra Control (Fuente: Astrid Esquivel Álvarez, 2014).

Las pinturas de Cobá pertenecen a la primera tradición colorística de la zona maya, la cual se inició en el periodo Clásico, y suele insertarse entre los años $200 \mathrm{~d}$. C. y 900 d. C. (Magaloni 1996:174). Los artistas de este y otros sitios experimentaron con el colorante obtenido de las hojas y ramas frescas de la Indigofera suffruticosa, combinado con algún filosilicato (normalmente, paligorskita, atapulgita o saponita), para dar lugar a los ricos tonos verde azulados que caracterizan a los murales de la zona (Figura 2). Correspondientemente, los resultados de XRD indican la presencia de un aluminosilicato de magnesio, pero en cuanto a la especie cristalina, no fue posible definirla a causa de la reducida cantidad de material con el que se contaba para el estudio (Figura 3). Asimismo, en el análisis con reflectancia total atenuada (IR-ATR) se distinguió un ondulamiento cercano a la región que va de los 3400 a los $3200 \mathrm{~cm}$, la misma en que se ubica el índigo natural; es decir, existe la posibilidad de que estos fragmentos estén elaborados con tales materiales (Figura 4).

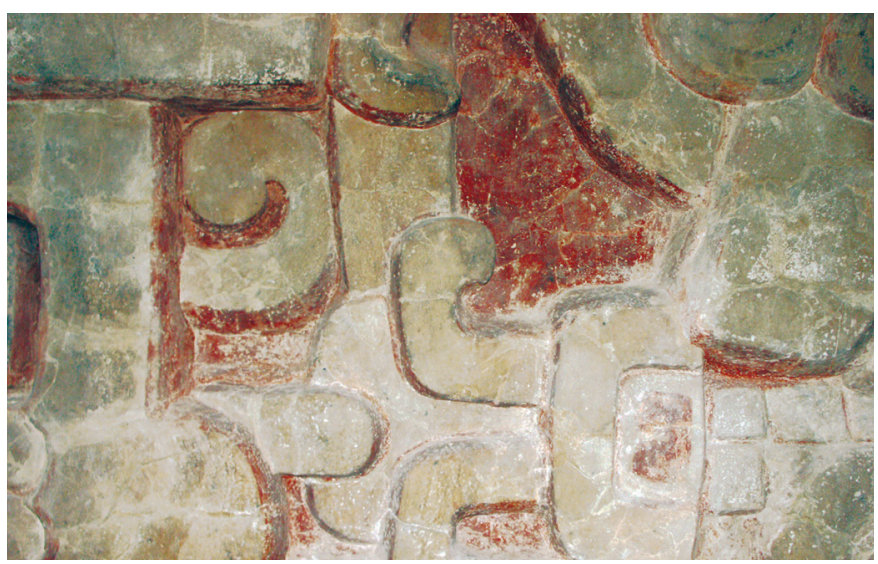

FIGURA 4. Espectro IR-ATR de la muestra Control Maya (Fuente: Astrid Esquivel Álvarez, 2014). 


\section{Estado de conservación}

La superficie de los fragmentos de pintura mural prehispánica empleados en esta investigación presentaba varios efectos de alteración (Figura 2), todos ellos relacionados con la estabilidad física de los materiales. Un ángulo de contacto igual a cero indicó un sustrato altamente poroso, en tanto que una microdureza de 89.07 HV y un factor de cohesión —obtenido a partir del test de la cinta Scotch ${ }^{\circledR}$ equivalente a $0.0124 \mathrm{~g} / \mathrm{cm}^{2}$ demostraron que la resistencia mecánica era pobre. Al respecto, Magaloni (1996:170) menciona que tanto la capa pictórica como los enlucidos de las pinturas del Grupo Técnico IV de la Zona Arqueológica de Cobá son fácilmente disgregables y deleznables, producto, quizá, del uso de materiales de baja calidad. Por otro lado, el análisis a la gota para la identificación de aniones asociados a depósitos salinos desveló la presencia de sales carbonatadas y sulfatadas. Se cree que las sales agudizan los efectos antes mencionados. Como un proceso de desalinización previo a la consolidación de los fragmentos supondría pérdida de material original en la capa pictórica, en este caso, la parte más significativa de estas y otras piezas, se optó por no llevarlo a cabo en esta ocasión.

\section{Resultados de la experimentación}

El análisis de los resultados obtenidos de este experimento fue más complicado de estudiar, dada la cantidad de variables que incidieron en el proceso. Además del porcentaje de HR, la cantidad de dióxido de carbono y la tasa de evaporación del disolvente, se vio que la técnica pictórica, la porosidad, el método de aplicación y la concentración del producto influyeron en el desempeño de Nanorestore ${ }^{\circledR}$, al menos en términos de efectos en superficie.

Primeramente, se advirtió una clara mejora en la resistencia mecánica de todas las muestras, así como una disminución en el efecto de pulverulencia en todos los fragmentos. También se reparó en la aparición de velos blanquecinos y otros depósitos del material, principalmente en fisuras y huecos (Figura 5). Se cree que estos efectos tuvieron su origen en el método de aplicación: los pinceles introducían gran cantidad de líquido de una sola intención y saturaban el sustrato, por lo que una buena parte del consolidante permanecía en la superficie de la capa pictórica, donde se secaba formando, casi siempre, velos o aglomerados. El efecto fue más notorio en ambientes secos, donde el disolvente se evaporaba más velozmente.

Al analizar las muestras bajo la lente del SEM (Figura 6) se comprobó que en todas, el producto exhibía cristales de formas ovaladas similares a los obtenidos de la muestra que estuvo dentro de la cámara 1, durante el experimento anterior, y no se encontraron cristales en forma de cubo.

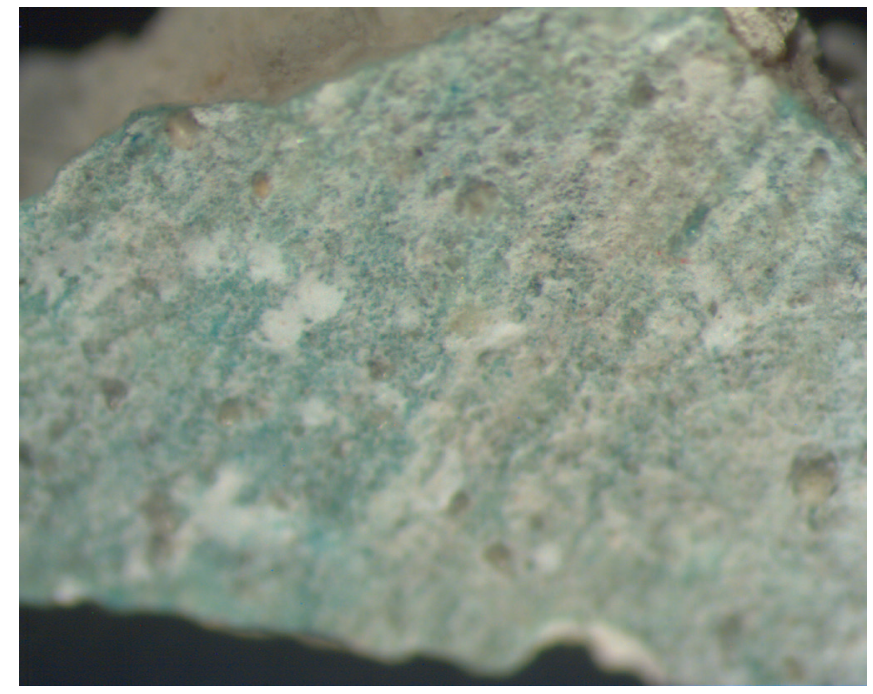

FIGURA 5. Muestra M2 al cabo de seis aplicaciones del consolidante (Fuente: Astrid Esquivel Álvarez, 2014; cortesía CNCPC: INAH).

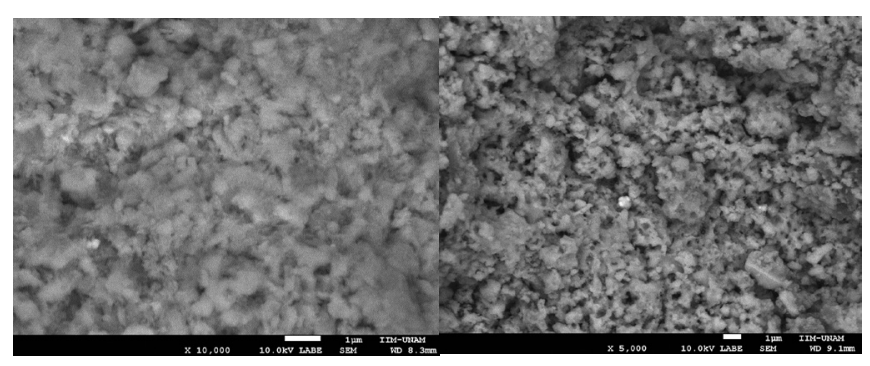

M1 $(10,000 X)$

M2 (5,000X)

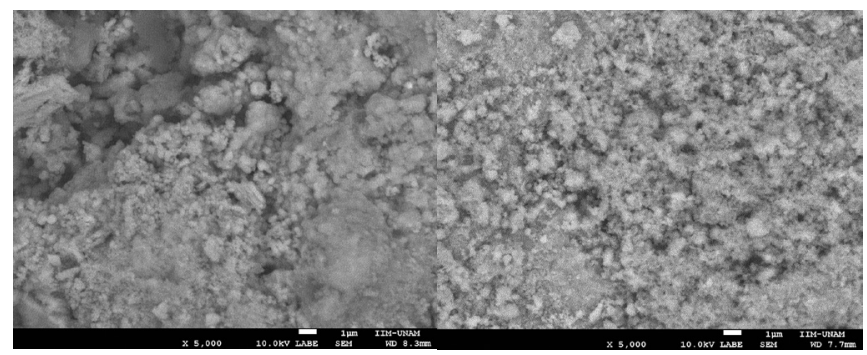

M3 $(5,000 \mathrm{X})$

M4 (5,000X)

FIGURA 6. Muestras 1-4 al cabo de 12 aplicaciones (Fuente: Astrid Esquivel Álvarez, 2014).

Se cree que esto fue consecuencia de los cambios de presión ejercida por las moléculas de gas, en función de las características de la superficie expuesta y del tiempo. En el caso anterior, el área de las nanopartículas era más homogénea, por lo que la fuerza promedio impresa por el gas sobre la superficie del sólido fue constante, en tanto que en este caso ocurrió algo distinto. Por otra parte, en aquellas muestras sometidas a porcentajes de humedad bajos (M1 y M3), la distribución del consolidante fue más heterogénea, y desigual respecto de los otros casos (M2 y M4), es decir, el uso de papetas de agua destilada no garantizó que el producto se reordenara. Todas estas observaciones pueden constatarse en la Figura 6. 
El estudio con XRD fue especialmente complicado, dada la semejanza entre las especies cristalinas detectadas desde un inicio y las que se obtuvieron a partir del consolidante. En términos generales, fue notable que los ambientes con altas humedades relativas (cámara 2), junto con una mayor cantidad de $\mathrm{CO}_{2}$ (cámara 4), favorecieron el proceso de carbonatación, mientras que las muestras expuestas a porcentajes de humedad bajos (cámara 3) y una mayor cantidad de $\mathrm{CO}_{2}$ (cámara 1) exhibían una carbonatación incompleta o pobre. Por ello no resultó extraño que la muestra $M 1$ conservara una porción de portlandita, lo que indicó que el consolidante seguía reactivo, ni que en las muestras M2 y M3 se haya encontrado vaterita y aragonita, en tanto que la M4 fue la única donde el consolidante carbonató completamente. Cabe resaltar que es muy probable que la porosidad del sustrato haya determinado la cantidad de $\mathrm{CO}_{2}$ que ingresaba en los poros de las muestras, lo que pone de manifiesto que las características del soporte también inciden en las reacciones del consolidante. Las observaciones descritas se sintetizan en la Figura 7.

\begin{tabular}{|c|c|c|}
\hline \multicolumn{3}{|c|}{ DRX } \\
\hline & 6 aplicaciones & 12 aplicaciones \\
\hline $\begin{array}{c}\text { Cámara } 1 \\
30 \% \mathrm{HR} \\
\mathrm{CO}_{2} 0.0 \% / \mathrm{Vol}\end{array}$ & $\begin{array}{c}\text { calcita, cuarzo, } \\
\text { aragonita, } \\
\text { portlandita }\end{array}$ & $\begin{array}{c}\text { calcita, cuarzo, } \\
\text { aragonita, } \\
\text { portlandita }\end{array}$ \\
\hline $\begin{array}{c}\text { Cámara } 2 \\
80 \% \mathrm{HR} \\
\mathrm{CO}_{2} 0.0 \% / \mathrm{Vol}\end{array}$ & $\begin{array}{c}\text { calcita, cuarzo, } \\
\text { aragonita, vaterita }\end{array}$ & $\begin{array}{c}\text { calcita, cuarzo, } \\
\text { aragonita, vaterita }\end{array}$ \\
\hline $\begin{array}{c}\text { Cámara } 3 \\
30 \% \mathrm{HR}, \\
\mathrm{CO}_{2} 1.0 \% / \mathrm{Vol}\end{array}$ & $\begin{array}{l}\text { calcita, cuarzo, } \\
\text { aragonita, vaterita }\end{array}$ & $\begin{array}{c}\text { calcita, cuarzo, } \\
\text { aragonita, vaterita }\end{array}$ \\
\hline $\begin{array}{c}\text { Cámara } 4 \\
80 \% \mathrm{HR}, \\
\mathrm{CO}_{2} 1.0 \% / \mathrm{Vol}\end{array}$ & $\begin{array}{l}\text { calcita, cuarzo, } \\
\text { aragonita }\end{array}$ & $\begin{array}{l}\text { calcita, cuarzo, } \\
\text { aragonita }\end{array}$ \\
\hline
\end{tabular}

FIGURA 7. Resultados de XRD a mitad y al final del tratamiento de consolidación (Fuente: Astrid Esquivel Álvarez, 2014).

Asimismo, el estudio de parámetros de afinidad superficial derivados del ángulo de contacto mostró que algunas propiedades, como la capilaridad y la capacidad de absorción de agua, sufrieron modificaciones significativas. Inicialmente, los sustratos presentaban un ángulo de contacto equivalente a cero, es decir, un mojado perfecto, indicativo de alta porosidad y de una resistencia mecánica baja. A medida que avanzaba el tratamiento, las muestras exhibían, por una parte, un aumento progresivo en el ángulo de contacto y, por la otra, una penetración en el sustrato más lenta. Esto fue consecuencia de la manera en la que el consolidante precipitó en superficie, así como de su tendencia a formar partículas grandes. No obstante, se observó que las muestras seguían siendo permeables, esto es, había continuidad en ciclos de absorción y desorción de humedad ambiental. Lo anterior se traduce como una ventaja sobre otros productos, como los polímeros sintéticos, bien conocidos por anular casi por completo esta cualidad.

En cuanto a los resultados de microdureza Vickers, todas las muestras, sin importar las condiciones a las cuales se expusieron, manifestaron un aumento significativo en el fortalecimiento de la resistencia mecánica en superficie. Partiendo del hecho de que la dureza está parcialmente determinada por enlaces intermoleculares fuertes, se llegó a la conclusión de que la semejanza entre los materiales constitutivos de las muestras y el consolidante hizo posible establecer una unión firme y estable. En otras palabras, el aumento de resistencia también estuvo condicionado por la afinidad que existía entre el consolidante y el sustrato.

Con el test de la cinta Scotch ${ }^{\circledR}$ fue evidente que el tratamiento de consolidación con nanopartículas de hidróxido de calcio tuvo un efecto remarcable en el incremento de cohesión de la capa pictórica de todas las muestras, pues disminuyó significativamente la pérdida de material original; en cuanto a la del pigmento, pasó, como se observa en la Figura 8 , de $0.0124 \mathrm{~g} / \mathrm{cm}^{2}$ (antes del tratamiento) a $0.0086 \mathrm{~g} / \mathrm{cm}^{2}$ (después del tratamiento), logrando una disminución de $70 \%$ en ese valor. Con base en lo anterior se concluyó que tanto la concentración del consolidante como la cantidad y frecuencia con la que se suministró el producto determinaron la ganancia de resistencia, y que el aumento de cohesión no dependió directamente de las condiciones a las que se sometieron las muestras.

Finalmente, un análisis de propiedades de apariencia y color demostró que, en términos generales, la consolidación de la capa pictórica provocó la disminución progresiva de la saturación, el tono y la textura; no así el

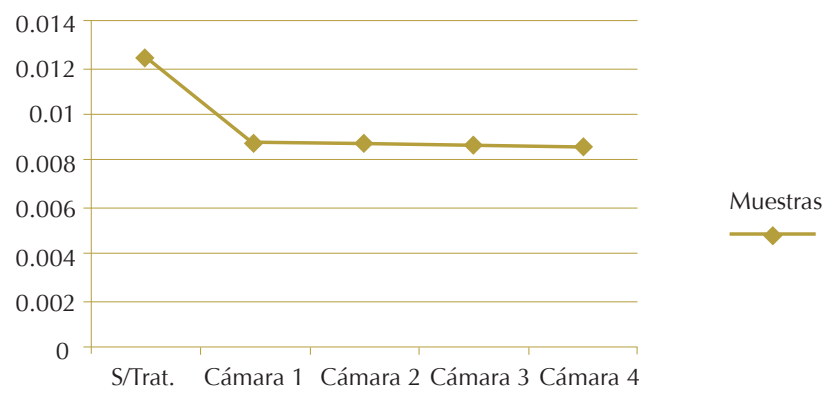

FIGURA 8. Test de la cinta Scotch ${ }^{\circ}$. Muestras sin tratamiento y después de haber concluido el tratamiento (Fuente: Astrid Esquivel Álvarez, 2014) 
brillo y la luminosidad —que se incrementaron conforme pasaba el tiempo-; por razón de que ésta se traduce como la cercanía de un color al blanco, concepto muchas veces asociado a la brillantez, no sorprende que los valores aumentaran a medida que se aplicaba el material. El fenómeno es equivalente y contrario para el caso de los otros atributos del color, pues se entiende que la adición de blanco a un color determinado se traduce en pérdida de saturación (es decir, provoca una disminución en la pureza del color) y modifica el tono (altera el estímulo cromático original) al ejecutar el tratamiento. El efecto es una consecuencia del número de aplicaciones y de la concentración del producto, por lo que conviene iniciar el tratamiento con una concentración baja.

\section{Conclusiones}

Estos dos experimentos confirman la hipótesis planteada al inicio del trabajo de investigación: las nanopartículas dehidróxido de calcio Nanorestore ${ }^{\circledR}$ son materiales más delicados de lo que se creía, y su desempeño depende en mucha parte de la manera en que se fabrican, de las características del sustrato al cual se aplican y de las condiciones del medio ambiente. Fue patente que, de haber humedad condensada en la superficie del mural o al interior de los poros, la consolidación no será efectiva, pues el producto reaccionará con el agua superficial sin poder adentrarse en el sustrato debilitado. Esto lleva a resolver que no es recomendable en un ambiente en que la $\mathrm{HR}$ supere $80 \%$, o donde la humedad se condense en la superficie. Sin embargo, en un ambiente donde la humedad sea menor a $30 \%$ se corre el riesgo de obtener especies inestables o de dejar al material reactivo. Asimismo, se debe procurar que el nivel de $\mathrm{CO}_{2}$ supere al habitual a lo largo del tratamiento y unos cuantos días después de que se haya concluido.

Se cree que la aplicación del consolidante a intervalos más separados (quizá cada cuatro o cinco días, en lugar de tres) beneficiaría la producción de calcita, muy conveniente para aquellos sustratos con ésta como compuesto principal. Se piensa, sin embargo, que en casos donde haya otras especies cristalinas, como la aragonita, este punto no es imperativo.

Por otra parte, el aumento en la cohesión y en la resistencia mecánica de todas las muestras fue muy evidente. Se llegó a la conclusión de que este aumento no está determinado por las condiciones a las que se someten las muestras, sino, más bien, se relaciona con la concentración del producto, la cantidad y frecuencia con la que se suministra. Si éstos son los casos, se recomiendan proporciones bajas para hacer de la aplicación un proceso controlable y paulatino.

De igual manera, las alteraciones cromáticas, consecuencia de la formación de velos blanquecinos y depósitos en huecos o fisuras, resultaron de la tendencia de Nanorestore ${ }^{\circledR}$ a agruparse en cúmulos y formar cristales grandes. Es muy posible que esta característica se deba a los materiales y métodos empleados en la fabricación del producto, o bien que obedezca al método de aplicación, que involucra el uso de pinceles, papel japonés y papetas de pulpa de celulosa.

Respecto de este problema, existen varias soluciones. Podría llevarse a cabo una serie de pruebas con nanocales sintetizadas en laboratorios locales; variar el método de aplicación a otro que no involucre las herramientas y materiales mencionados, como, por ejemplo, aspersiones y nebulizaciones, para aplicar tanto el consolidante como el agua.

Se observó también, por último, que el éxito del tratamiento depende en parte de la afinidad existente entre el consolidante y el sustrato. Por ejemplo, cuando las pinturas murales estén constituidas por cales dolomíticas o contengan altos contenidos de silicatos, la efectividad del material estará limitada, pues no podrá establecer con tanta facilidad uniones intermoleculares.

\section{Agradecimientos}

Agradecemos a los doctores Diana Magaloni y Antonio Saborit, del Museo Nacional de Antropología (MNA), por el apoyo a la investigación aplicada a la restauración en el MNA-INAH. A los doctores Pedro Bosch, Enrique Lima, Omar Novelo, Gabriel Lara, y a la maestra Adriana Tejeda, todos ellos del Instituto de Investigaciones en Materiales (IIM-UNAM), por su colaboración en el análisis e interpretación de muestras. A las doctoras Valerie Magar, Isabel Villaseñor y Nora Pérez, y a las licenciadas María del Carmen Castro e Irlanda Fragoso, de la Coordinación Nacional de Conservación del Patrimonio Cultural (CNCPC) del INAH, por los equipos y las facilidades otorgadas.

\section{Referencias}

Alton,Wade F. y Richard Mattox

1963 Elementos de la Cristalografía y Mineralogía, Barcelona, Omega.

Ambrosi, Moira, Luigi Dei, Rodorico Giorgi, Chiara Neto y Piero Baglioni

2001a "Colloidal Particles of $\mathrm{Ca}(\mathrm{OH}) 2$ : Properties and Application to Restoration of Frescoes", Journal of the American Chemical Society, 17 (14):4251-4255.

2001b "Stable Dispersions of $\mathrm{Ca}(\mathrm{OH}) 2$ in Aliphatic Alcohols: Properties and Application in Cultural Heritage Conservation", Trends in Colloid Interface Science, 15:68-72. ASTM

2011 "ASTM E38411E1", Standard Test Method for Knoop and Vickers Hardness of Materials, American Society for Testing Materials International.

Baglioni, Piero y Rodorico Giorgi

2006a "Soft and Hard Nanomaterials for Restoration and Conservation of Cultural Heritage", Soft Matter, 2 (4):293303. 
Baglioni, Piero, Ramón Vargas, David Chelazzi, Marinés Colón, Alice Desprat y Rodorico Giorgi

2006b "The Maya Site of Calakmul: in Situ Preservation of wall Paintings and Limestone Using Nanotechnology", Proceedings of the IIC Congress: The Object in Context: Crossing Conservation Boundaries, 162-169.

Baglioni, Piero, Rodorico Giorgi y Luigi Dei 2009 "Soft Condensed Matter for the Conservation of Cultural Heritage", Comptes Rendus Chimie, 12 (1-2):61-69.

Barthlemy, David 1997 Mineralogy Database [página web], documento electrónico disponible en [http://webmineral.com], consultado el 20 de diciembre de 2015.

Benavides, Alfonso

1981 Informe de actividades de la temporada 1981 del Proyecto Cobá, México, documento mecanoescrito inédito, México, Archivo Técnico de la Coordinación Nacional de Arqueología-INAH.

Castro, María del Carmen

2009 Informe técnico parcial de los trabajos de conservación y restauración de la caja de agua, temporada 20072009, México, documento mecanoescrito disponible en Archivo Técnico, Coordinación Nacional de ArqueologíaINAH.

Castro, María del Carmen, Yareli Jáidar, Piero Baglioni y Rodorico Giorgi

2013 "Nanotecnología aplicada a la consolidación de pinturas murales. Nanopartículas de hidróxido de calcio, $\mathrm{Ca}(\mathrm{OH}) 2^{\prime \prime}$, en Luis Barba e Isabel Villaseñor (eds.), La cal. Historia, propiedades y usos, México, IIE-UNAM, 235-258.

Chelazzi, David, Giovanna Poggi, Yareli Jáidar, Nicola Toccafondi, Rodorico Giorgi y Piero Baglioni

2013 "Hydroxide Nanoparticles for Cultural Heritage: Consolidation and Protection of wall Paintings and Carbonate Materials", Journal of Colloid and Interface Science, 392 (1):42-49.

D'Armada, Paul y Elizabeth Hirst

2012 "Nano-lime for Consolidation of Plaster and Stone", Journal of Architectural Conservation, 18 (1):63-80.

Dei, Luigi

2004 Nanotecnologie per la conservazione di opere d'arte. Propiet'a ed applicazioni dei minerali alla nanoscale, Università degli Studi di Firenze (CSGI), documento electrónico disponible en [http://www.socminpet.it/GNM/GNM_04/ Dei.pdf], consultado en enero de 2016.

Dei, Luigi, Bruno Radicati y Barbara Salvadori

2005 "Sperimentazione di un consolidante a base di idrossido di calcio nanofasico sugli affreschi della cappella del Podestà al Museo del Bargello di Firenze: aspetti chimicofisici e prove di colore", Atti del Convegno di Bressanone, 293-302.

Dietrich, Richard y Brian Skinner

1979 Rocks and Rock Minerals, Nueva York, John Wiley and Sons.
Doménech-Carbó, María Teresa, Laura Osete-Cortina, Antonio Doménech-Carbó, María Luisa Vázquez y Cristina Vidal-Loren-

ZO

2014 "Identification of Indigoid Compounds Present in Archaeological Maya Blue by Pyrolysis-silylation-gas Chromatography-mass spectrometry", Journal of Analytical and Applied Pyrolysis, 105:355-362.

Duch-Gary, Jorge

1991 Fisiografía del estado de Yucatan, México, UAdY.

Esquivel Álvarez, Astrid

2014 Factores que influyen en los procesos de carbonatación de nanopartículas de hidróxido de calcio aplicados a pinturas murales prehispánicas con problemas de cohesión y pulverulencia en su capa pictórica, tesis de licenciatura en conservación y restauración de patrimonio cultural mueble, México, ENCRYM-INAH.

Folan, William

1977 "El Sacbé Cobá-Ixil: un camino maya del pasado", Nueva Antropología, 2 (6):31-42.

Friedman, Hershel

1997 The Mineral and Gemstone Kingdom [página web], documento electrónico disponible en [http://www.mine rals.net], consultado el 20 de diciembre de 2015.

García Solís, Claudia

2008 "Informe preliminar de la visita del Dr. Piero Baglioni a la Zona Arqueológica de Mayapán", México, Centro INAH-Yucatán, Coordinación Nacional de Conservación del Patrimonio Cultural-INAH.

2011 "Informe de los procesos de conservación de la pintura mural de los símbolos solares en la Zona Arqueológica de Mayapán", Yucatán, México, Centro INAH-Yucatán/ ENCRyM/Coordinación Nacional de Conservación del Patrimonio Cultural-INAH.

Giorgi Rodorico, Luigi Dei y Piero Baglioni 2000 "A New Method for Consolidation wall Paintings Based on Dispersions of Lime in Alcohol", Studies in Conservation, 45 (3):154-161.

Giorgi, Rodorico, Luigi Dei, Massimo Ceccato, Claudius Schettini y Piero Baglioni

2002 "Nanotechnologies for Conservation of Cultural Heritage: Paper and Canvas Deacidification", Journal of the American Chemical Society, 18 (21):8198-8203.

Giorgi, Rodorico, Michele Baglioni, Debora Berti y Piero Baglioni

2010 "New Methodologies for Conservation of Cultural Heritage: Micellar Solutions Microemulsions and Hydroxide Nanoparticles", Accounts of Chemical Research, 43 (6):695-704.

Grimaldi, Dulce

2008 "Informe de las pruebas de consolidación y limpieza de la pintura mural de Los Bebedores en Cholula, Puebla", México, Coordinación Nacional de Conservación del Patrimonio Cultural-INAH.

Gómez-Villalba, L. Stella, Paula López-Arce, Mónica Álvarez de Buergo y R. Fort

2011a "Structural Stability of a Colloidal Solution of 
$\mathrm{Ca}(\mathrm{OH})_{2}$ Nanocrystals exposed to high Relative Humidity Conditions", Applied Physics A, (104):1249-1254.

Gómez-Villalba, Luz Stella, Paula López-Arce y Rafael Fort 2012b "Nucleation of $\mathrm{CaCO}_{3}$ Polymorphs from a Colloidal Alcoholic Solution of $\mathrm{Ca}(\mathrm{OH})_{2}$ Nanocrystals Exposed to low Humidity Conditions", Applied Physics A, (106):213-217.

ICOMOS-ISCS

2010 Illustrated Glossary of Stone Deterioration Patterns, Paris, ICOMOS.

Jáidar Benavides, Yareli

2014 Nanotechnology Applied to the Conservation and Restoration of Pre-hispanic Cultural Heritage, tesis doctoral en ciencia aplicada a la restauración, Università degli Studi di Firenze.

López-Arce, Paula, Luz Stella Gómez-Villalba, Sagrario Martínez-Ramírez, Mónica Álvarez de Buergo y Rafael Fort

2011a "Influence of Relative Humidity on the Carbonation of Calcium Hydroxide Nanoparticles and the Formation of Calcium Carbonate Polymorphs", Powder Technology, (205):263-269.

Magaloni, Diana

1996 "Materiales y técnicas de la pintura mural maya", tesis de maestría en historia del arte, Facultad de Filosofía y Letras, FFYL-UNAM.

Mansoori, Ali

2004 Principles of Nanotechnology: Molecular-Based Study of Condensed Matter in Small Systems, Chicago, University of Chicago.

Montes-Hernández, Germán, F. Renard, N. Geofroy, L. Charlet y J. Pironon

2007 "Calcite Precipitation from $\mathrm{CO}_{2}-\mathrm{H}_{2} \mathrm{O}-\mathrm{Ca}(\mathrm{OH})_{2}$ Slurry Under high Pressure of $\mathrm{CO}_{2}$ ", Journal of Crystal Growth, 308 (1):228-236.

Montes-Hernández, Germán, Alejandro Fernández-Martínez, Laurent Charlet, D. Tisserand y François Renard

2008 "Textural Properties of Synthetic Nano-calcite Produced by Hydrothermal Carbonation of Calcium Hydroxide", Journal of Crystal Growth, (310):2946-2953.

Montes-Hernández, Germán y François Renard

2011 "Co-utilisation of Alkaline Solid waste and Compressed-or-supercritical $\mathrm{CO}_{2}$ to Produce Calcite and calcite/Se red Nanocomposite", The Journal of Supercritical Fluids, 56 (1):48-55.

Ogino, Takeshi, Toshio Suzuki y Kiyoshi Sawada 1987 "The Formation and Transformation Mechanism of Calcium Carbonate in Water", Geochimica et Cosmochimica Acta, 51 (10):2757-2767.
Rodgers, Sylvia

1988 "Consolidation/fixing/facing", The Paper Conservation Catalog, Washington D. C., AIC, 2-18.

Rodríguez-Blanco, Juan Diego, Sam Shaw y Liane Benning 2011 "The Kinetics and Mechanisms of Amorphous Calcium Carbonate Crystalization to Calcite", Nanoscale, (3):265-271.

Soldati, Aanalia, Dorrit Jacob, Ursula Wehrmeister y W. Hofmeister

2008 "Structural Characterization and Chemical Composition of Aragonite and Vaterite in Freshwater Cultured Pearls", Mineralogical Magazine, 72 (2):579-592.

Uibu, Mai, Olga Velts, y R. Kuusik 2010 "Developments in $\mathrm{CO}_{2}$ Mineral Carbonation of Oil Shale ash", Journal of Hazardous Materials, 174 (1-3):209214.

\section{Síntesis curriculares del/los autor/es}

\section{Astrid Esquivel Álvarez}

School of the Art Institute of Chicago (SAIC), Estados Unidos de América astridesquivel7@gmail.com

Licenciada en restauración (Escuela Nacional de Conservación, Restauración y Museografía, Instituto Nacional de Antropología e Historia [ENCRyM-INAH], México). Dedicó su tesis de licenciatura al estudio de la efectividad de nanoconsolidantes aplicados a pinturas murales prehispánicas. Actualmente cursa la maestría en ciencias de la conservación (School of the Art Institute of Chicago [SAIC], Escuela del Instituto de Arte de Chicago, EUA), donde enfoca sus estudios en el control climático de museos.

\section{Laura Filloy Nadal}

Museo Nacional de Antropología (MNA),

Instituto Nacional de Antropología e Historia (INAH), México

Ifilloy@yahoo.com

Licenciada en restauración (Escuela Nacional de Conservación, Restauración y Museografía del Instituto Nacional de Antropología e Historia [ENCRyM-INAH], México). Maestra y doctora en arqueología (Universite de Paris 1, Sorbone, Francia). Profesora de asignatura en la ENCRYM-INAH y en la Escuela Nacional de Antropología e Historia (ENAH), México. Cuenta con una trayectoria de más de 20 años como restauradora perito del Museo Nacional de Antropología (MNA-INAH), México.

Postulado/Submitted 11.12.15

Aceptado/Accepted 05.04.16

Publicado/Published 11.07.16 